\title{
Quantitative Approaches to Study Elemental Distribution on Fluid Catalytic Cracking Catalyst
}

\author{
YL Tang $^{1 *}$, Mehdi Allahverdi ${ }^{1}$ and Paul Diddams ${ }^{1}$ \\ 1. Johnson Matthey, Savannah, GA, USA. \\ * Corresponding author: yali.tang@matthey.com
}

The Fluid Catalytic Cracking (FCC) unit is one of the main conversion units in a refinery. It processes typically a vacuum gasoil or atmospheric residue hydrocarbon feed. These feeds contain contaminant transition metals that are deposited on the FCC catalyst. With the growth of shale oils usage in FCC units metal contaminants could become more problematic [1,2]. For example, feed iron poses a challenge and manifest itself in different ways. Every transition metal has different mechanisms how it poisons FCC catalyst: dissolving active components (vanadium), adding unwanted reactions (nickel) or forming a dense surface that makes the catalyst inaccessible (iron). Analyzing metal distributions on the catalyst surface plays an important role in understanding the deposition and mobility of the poisoning species.

In this study we obtained the Quantitative statistical data for the elemental distribution from hundreds to thousands of FCC catalyst particles in an equilibrium catalyst. This technique reveals the mobility of metals over FCC catalyst particles. This is the first time that combination of statistics with electron microscopy surface analysis has been applied to study metal migration in industrial catalysts (Figure 1). The high mobility of vanadium and immobility of nickel are confirmed. Surprisingly, feed iron shows to be highly mobile under FCC conditions (Figure 2), a fact that was previously unknown. This study also shows that the iron diffusion mechanism differs from vanadium: iron migration occurs as agglomerates via particle-particle collisions, whereas vanadium can diffuse via the gas phase. This discovery enables the development of separate metal traps to counter the poisonous effects of iron and can bring a substantial economic benefit for refiners using shale oil with notable Fe content.

\section{References:}

[1] B De Graaf et al., PTQ, Processing Shale Feedstocks (2014), p. 31.

[2] YL Tang et al., Microscopy and Microanalysis 17 (S2) (2011), p. 1378. 


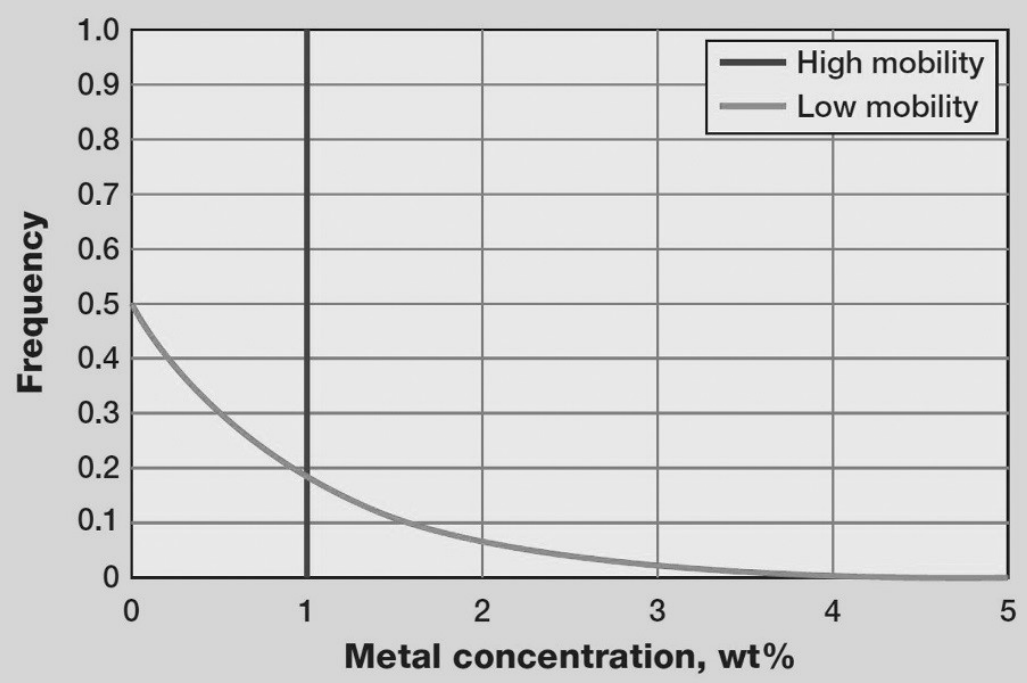

Figure 1. Distribution of surface metal over FCC ECAT for extremely high mobility (dark line) and extremely low mobility (grey line)

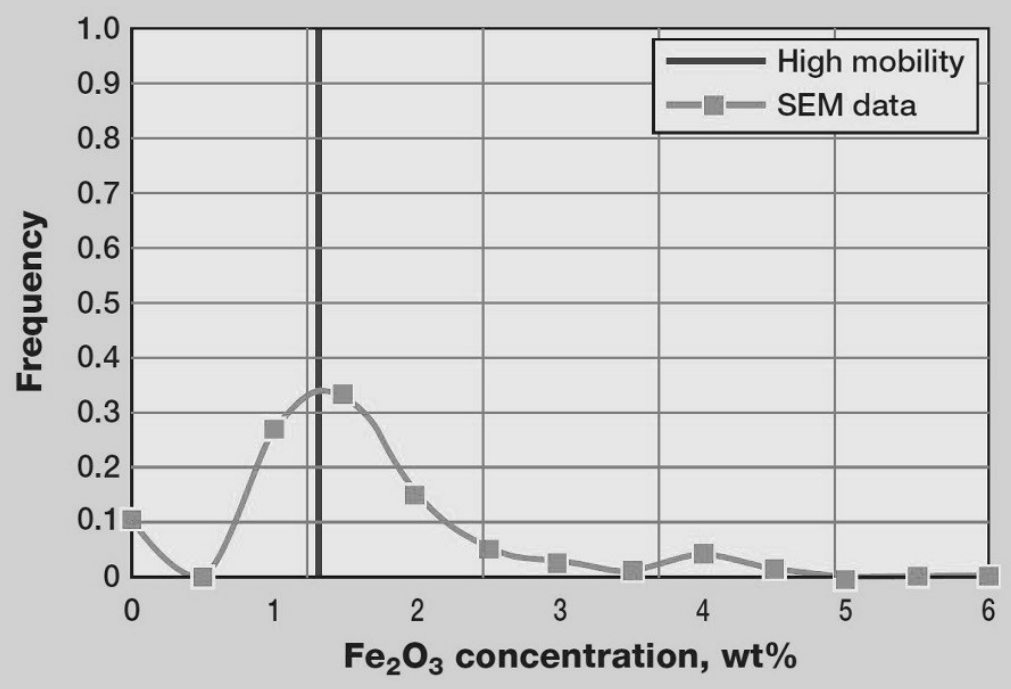

Figure 2. Distribution of $\mathrm{Fe}_{2} \mathrm{O}_{3}$ on the surface of FCC ECAT for extremely high mobility (dark line) and actual SEM/EDS data (grey line) 